\title{
Viewpoint
}

\section{Aging, Frailty and Complexity}

Marcel G. M. Olde Rikkert 1,2,3

1 Department Geriatric Medicine, Radboud University Medical Center, PO Box 9101, 6500HB Nijmegen, the Netherlands;

Email: marcel.olderikkert@radboudumc.nl

2 Radboudumc Alzheimer Center, Radboud University Medical Center, PO Box 9101, 6500HB Nijmegen, the Netherlands

3 Donders Institute Medical Neurosciences, Radboud University, PO Box 9101, $6500 \mathrm{HB}$ Nijmegen, the Netherlands

\section{ABSTRACT}

The big problems in aging research are of increasing complexity due to multicausality of chronic diseases and the numerous interactions between the multiple mechanisms of aging. The frailty concept is a first simple step forward as an integrative concept that sums up the deficits over multiple domains. However, for further understanding of the empirical data on this multicausality and interactions in aging, we need more sophisticated tools developed in complexity science. Group model building, computational modeling and network analyses are examples of tools that can push the frontier of gerontologic and geriatric research into a new era of putting the pieces together discovered in the detailed omics studies.

KEYWORDS: frailty; resilience; complexity science; computational modeling; group model building; network analyses

\section{INTRODUCTION}

Human beings are "complex systems" when one considers them in abstract terms as a multicomponent system (1), with many (feedback) interactions (2). In aging individuals these physiologic interactions also include the aging mechanisms, that are at least partly non-linear, (3)

\section{G Open Access}

Received: 03 December 2019 Accepted: 05 February 2020 Published: 12 February 2020

Copyright (c) 2020 by the author(s). Licensee Hapres, London, United Kingdom. This is an open access article distributed under the terms and conditions of Creative Commons Attribution 4.0 International License. history and environment dependent, (4) and of different temporal and spatial scales (5). The multicomponents can be mechanisms in cells and organs, but also in the environment (e.g., the patient's family) that have impact on the patients' physical and psychological aging and health. Together these five preconditions also are the criteria for defining a complex system according the Nobel prize winner and chemist Ilya Prigogine, as he formulated these in his probabilistic approach to the statistics of complex systems [1]. These criteria however have been embraced only recently by many other researchers in a quest for transcending the reductionist scientific quest to solve the big multicomponent societal problems, such as climate change, that we also face in dealing with the increasing burden of chronic diseases of older 
persons [2,3]. In fact the cumulative deficits model introduced successfully in aging research yields a solid body of evidence for the fact that the key topics of aging and age related diseases are related to multiple deficits and thus multicausality [4]. These multiple deficits of frailty are now starting to be studied as well by applying advanced network analyses together with big data analytics [5]. This altogether highlights the start of a new era in geriatric and gerontological research, in which embracing complexity and complexity science methods can bring fundamentally new insights in aging, as it did and still does in other scientific disciplines.

\section{COMPLEXITY SCIENCE}

Complexity science (CS) can bring a radical shift in how we think about many of the dynamic relationships and systems present in our world, in many scientific disciplines. A bibliometric review of complexity science in healthcare shows the increasing use of a complex systems lens to describe, understand and study aspects of healthcare over the past two and a half decades. This has been carried out successfully in health research across many countries, among which USA, UK, Australia and Canada are the leading nations in this research [6]. The rising number of publications suggests a global move to complement the reductionistic focus by a more integrated knowledge focus in medical research. Also the number of citations shows a steep rise over the last 40 years, with last year 228 biomedical publications with "complexity science" in the title, and a linear increase of citations from 1199 in 2000 to 9510 citations in 2018 (Web of Science search on December 3rd, 2019). This all provides evidences for the increasing relevance of complexity science for medical research, and other scientific domains [7,8].

\section{COMPLEXITY IN AGING}

Using tools from analysis of complex systems in mathematical and physical sciences, improves the understanding of aging biological systems (e.g., C. elegans) and older patients [9-13]. In physical systems (e.g., quantum mechanics), complexity is determined by factors such as the number of components, the number and quality of the interactions between them, the degree of scaling, and the structure of the smallest components. Biological networks are characterized by many of these factors, as well as by their highly dynamic change in organization, transformation, and degradation. Interactions occur at many levels on genetic, proteomic, metabolomic, cellular and organ scale by chemical and physical signaling [14]. In animals and humans, signaling also occurs at organism (e.g., by self-reflection) and social scale (e.g., parental advices), and thus signaling additionally occurs by all sorts of communicational and behavioral interactions. The multiple frailty related aging mechanisms that result in an increasing number of deficits add to these interactions. Together, all these multiscale interactions may occur simultaneously, and each component participates in several different signaling activities, 
making it rightly called a complex biological system. In our omics era, this even is a dazzling complexity.

Physical and chemical systems may more easily be reduced to units of complexity that can be experimented and modeled with. However, this basic mathematical and computational science of complex systems can also be translated to higher levels of complexity, multiple scale levels, including human aging physiology, but still needs to be tested for validity here. The increasing number of integrative computational modeling studies on complex chronic health problems such as obesity and diabetes [15], are an excellent example of the added value of CS methods, and also hold great promises for other threatening diseases such as Alzheimer's disease.

\section{FROM SIMPLE TO COMPLEX}

A frequently used approach to understand complexity is to start a qualitative approach of building multicausal qualitative models by mind mapping or the more sophisticated group based model building methodology. This starts with conceptually simple examples of interactions and gradually adds systems (or agents) that introduce new levels of interaction complexity [16]. Differences in time scales and spatial scales however limit what can be taken into account together at this first CS stage, which can be detailed with empirical network analyses and computational modeling. As more data on interactions are introduced, it becomes quickly clear where experimentation options end, and where new methodologies are needed to understand the system as a whole in terms of the functional details of individual components.

Notwithstanding this, the clues to improved understanding of many human diseases, including cancer, diabetes, chronic inflammatory diseases, and neurodegenerative disorders, lie in understanding the changed functioning (and malfunctioning) of interactions between biological components [17,18]. Often malfunctioning of a single organ (or organ part) does not cause serious problems due to redundancy in the physiological networks, but the combined effects of multiple malfunctioning components of an interacting network of organs are substantial, and life threatening. For example, hippocampal and prefrontal cortex atrophy are often seen together with white matter lesions as malfunctioning components or nodes in a dementia pathophysiology network, only together causing cognition and functional performance to deteriorate in daily living. An understanding of how individual (sub)components function is helpful, but not enough to understand the whole disease severity and the individually emerging disease presentations. This means that reducing the research focus to smaller and smaller components, has limits in understanding individual aging individuals and their huge variation present in clinical practice. Precision medicine, with a focus on genetic, proteomic and metabolomic phenotyping, will not be able to forecast treatment effects in complex 
diseases that are determined also by relevant interactions at a higher scale level. This also requires complexity science's multiscale modeling and predictions at a higher level. Biology of aging can be characterized by the changes over time at different hierarchically organized levels-namely molecular, cellular, physiological, and functional levels. Detailed knowledge of these interconnected changes together with their reactions following pathophysiological stressors is needed to understand the heterogeneous biology and functional performance outcomes in older persons [19]. Ongoing progress in big data handling may allow automated phenotyping in the near future to measure as many age-dependent phenotypes as possible within individuals over time and using those highdimensional phenotypic data to construct dynamic networks that facilitate aging and disease to be studied with sufficient integration and rigor [20].

\section{CONCLUSION}

In medicine, it is only the biology of aging together with the context of the whole person, that may finally allow to understand pathophysiology, symptoms and loss of autonomy in an older patient. Detailed knowledge of pathophysiology helps to answer this question, but still requires integration of the different parts with the case specific environmental knowledge. On the other hand, knowledge of the global view itself, and the knowledge of complex signaling networks, may also improve the molecular understanding of the interactions at micro levels, as for example was nicely shown for the study on the molecular genetic and cellular effects of loneliness [21,22]. Therefore, this new era of applying complexity science methods can help us make a new jump in understanding frailty, resilience and aging.

\section{CONFLICTS OF INTEREST}

The author declares to have no conflicts of interest.

\section{REFERENCES}

1. Prigogine I, Sengers I, Toffler A. Order out of chaos: man's new dialogue with nature. Brooklyn (NY, US): Verso; 2017.

2. Boulton JG, Llen PM, Bowman C. Embracing Complexity. Oxford (UK): Oxford Univ Press; 2015.

3. Thurner S, Hanel R, Klimek P. Introduction to the theory of complex systems. Oxford (UK): Oxford Univ Press; 2018.

4. Yarnall AJ, Sayer AA, Clegg A, Rockwood K, Parker S, Hindle JV. New horizons in multimorbidity in older adults. Age Ageing. 2017;46(6):882-8. doi: 10.1093/ageing/afx150

5. Rutenberg AD, Mitnitski AB, Farrell SG, Rockwood K. Unifying aging and frailty through complex dynamical networks. Exp Gerontol. 2018;107:126-9. doi: 10.1016/j.exger.2017.08.027 
6. Churruca K, Pomare C, Ellis LA, Long JC, Braithwaite J. The influence of complexity: a bibliometric analysis of complexity science in healthcare. BMJ Open. 2019;9(3):e027308.

7. van Nes EH, Arani BMS, Staal A, van der Bolt B, Flores BM, Bathiany S, et al. What Do You Mean, 'Tipping Point'? Trends Ecol Evol. 2016;31:902-4. doi: 10.1016/j.tree.2016.09.011

8. Battiston S, Farmer JD, Flache A, Garlaschelli D, Haldane AG, Heesterbeek H, et al. Complex systems. Complexity theory and financial regulation. Science. 2016;351(6275):818-9. doi: 10.1126/science.aad0299

9. Olde Rikkert MG, Dakos V, Buchman TG, Boer Rd, Glass L, Cramer AO, et al. Slowing Down of Recovery as Generic Risk Marker for Acute Severity Transitions in Chronic Diseases. Crit Care Med. 2016;44:601-6. doi: 10.1097/CCM.0000000000001564

10. Gijzel SMW, van de Leemput IA, Scheffer M, Roppolo M, Olde Rikkert MGM, Melis RJF. Dynamical Resilience Indicators in Time Series of Self-Rated Health Correspond to Frailty Levels in Older Adults. J Gerontol A Biol Sci Med Sci. 2017;72:991-6. doi: 10.1093/gerona/glx065

11. Gijzel SMW, van de Leemput IA, Scheffer M, van Bon GEA, Weerdesteyn V, Eijsvogels TMH, et al. Dynamical indicators of resilience in postural balance time series are related to successful aging in high-functioning older adults. J Gerontol A Biol Sci Med Sci. 2019;74:1119-26. doi: 10.1093/gerona/gly170

12. Gijzel SMW, Whitson HE, van de Leemput IA, Scheffer M, van Asselt D, Rector JL, et al. Resilience in Clinical Care: Getting a Grip on the Recovery Potential of Older Adults. J Am Geriatr Soc. 2019;67:2650-7. doi: 10.1111/jgs.16149

13. Scheffer M, Bolhuis JE, Borsboom D, Buchman TG, Gijzel SMW, Goulson D, et al. Quantifying resilience of humans and other animals. Proc Natl Acad Sci U S A. 2018;115(47):11883-90. doi: 10.1073/pnas.1810630115

14. Weng G, Bhalla US, Iyengar R. Complexity in biological signaling systems. Science. 1999;284:92-6.

15. Appel JM, Fullerton K, Hennessy E, Korn AR, Tovar A, Allender S, et al. Design and methods of Shape Up Under 5: Integration of systems science and community-engaged research to prevent early childhood obesity. PLoS One. 2019;14(8):e0220169. doi: 10.1371/journal.pone.0220169

16. Hovmand PS, Andersen DF, Rouwette E, Richardson GP, Rux K, Calhoun A. Group Model-Building 'scripts' as a collaborative planning tool. Syst Res. 2012;29:179-93.

17. Kenzie ES, Parks EL, Bigler ED, Wright DW, Lim MM, Chesnutt JC, et al. The dynamics of concussion: Mapping pathophysiology, persistence, and recovery with Causal-Loop diagramming. Front Neurol. 2018;9:203.

18. Te Velde AA, Bezema T, van Kampen AH, Kraneveld AD, 't Hart BA, van Middendorp H, et al. Embracing Complexity beyond Systems Medicine: A New Approach to Chronic Immune Disorders. Front Immunol. 2016;7:587. doi: 10.3389/fimmu.2016.00587

19. Ferrucci L, Levine ME, Kuo PL, Simonsick EM. Time and the Metrics of Aging. Circ Res. 2018;123:740-4. doi: 10.1161/CIRCRESAHA.118.312816 
20. Freund A. Untangling Aging Using Dynamic, Organism-Level Phenotypic Networks. Cell Sys. 2019;8:172-81. https://doi.org/10.1016/j.cels.2019.02.005

21. Miller G. Social neuroscience. Why loneliness is hazardous to your health. Science. 2011;331(6014):138-40. doi: 10.1126/science.331.6014.138

22. Canli T, Yu L, Yu X, Zhao H, Fleischman D, Wilson RS, et al. Loneliness 5 years ante-mortem is associated with disease-related differential gene expression in postmortem dorsolateral prefrontal cortex. Transl Psychiatry. 2018;10:2. doi: 10.1038/s41398-017-0086-2

How to cite this article:

Olde Rikkert MGM. Aging, Frailty and Complexity. Adv Geriatr Med Res. 2020;2(2):e200008. https://doi.org/10.20900/agmr20200008 